\title{
INTERCORRÊNCIAS OBSTÉTRICAS NA ADOLESCÊNCIA: UMA REVISÃO \\ NARRATIVA DA LITERATURA
}

\section{Mayra Cristina Cavalcante Campos ${ }^{1}$; Ana Clara da Silva Beltrão ${ }^{1}$; Beatriz Albuquerque Bomfim $^{\mathbf{1}}$; Carlos Arthur Marinho da Silva Beltrão ${ }^{\mathbf{1}}$; Rafaela Cruz de Oliveira ${ }^{\mathbf{1}}$; Sofia

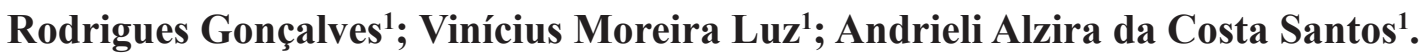

${ }^{1}$ Acadêmico de Medicina, Universidade do Estado do Amazonas (UEA), Manaus, Amazonas.

DOI: 10.47094/IICNNESP.2021/147

\section{RESUMO}

Introdução: A gravidez na adolescência pode ser considerada de risco e é um problema de saúde pública, sendo determinante para o aumento da morbimortalidade materna e neonatal e para o desenvolvimento de problemas psicossociais. Objetivo: Discorrer sobre as intercorrências obstétricas mais comuns em mulheres adolescentes. Metodologia: Trata-se de um artigo de revisão narrativa da literatura, que utilizou como fonte para a pesquisa bibliográfica as bases de dados eletrônicas SciELO e PubMed. Realizou-se a seleção de artigos publicados em português, no período de 2010 a 2021, utilizando como descritores "gravidez na adolescência" e "complicações na gravidez". Fundamentação teórica: Entre as intercorrências obstétricas comuns na gestação na adolescência, destacam-se as doenças hipertensivas da gestação, síndromes hemorrágicas, infecção urinária, abortos, baixo peso ao nascer e prematuridade. Considerações finais: Com a realização do pré-natal adequado, por uma equipe multidisciplinar, tais complicações obstétricas podem ser prevenidas.

PALAVRAS-CHAVE: Mulheres adolescentes. Complicações gestacionais. Mortalidade materna. ÁREA TEMÁTICA: Outros.

\section{INTRODUÇÃO}

No Brasil, para fins jurídicos, é considerado adolescente a pessoa de 12 a 18 anos, segundo o artigo $2^{\circ}$ do Estatuto da Criança e do Adolescente (ECA), Lei no 8069, de 1990. Já para o Ministério da Saúde, a adolescência compreende a faixa etária entre 10 e 24 anos e para a Organização Mundial da Saúde é a fase entre 10 e 19 anos (EISENSTEIN, 2005). Independente do período cronológico exato, é na fase da adolescência que a pessoa passa pelas maiores transformações anatômicas, fisiológicas, mentais e sociais, sendo um período que agrega inúmeros aprendizados, muito crescimento e desenvolvimento pessoal (MARTINS et al, 2011; OYAMADA et al, 2014).

Segundo Dias e Teixeira (2010), a gestação na adolescência se tornou um fenômeno evidente com o aumento da proporção de nascimentos de filhos de mulheres com idade inferior a 20 anos. Pelo fato de as adolescentes ainda apresentarem imaturidades físicas e psicológicas e estarem em maior vulnerabilidade social, a gravidez nesta fase passou a ser considerada de risco e um problema 
de saúde pública, sendo fator determinante para o aumento da morbimortalidade materna e neonatal e para o desenvolvimento de problemas psicossociais e econômicos (OLIVEIRA et al., 2010; SILVA et al., 2021).

Para a ginecologia e obstetrícia, muitas são as possíveis intercorrências que podem se apresentar durante uma gestação na adolescência, entre elas, pode-se citar: síndrome hipertensiva da gravidez, anemia, infecção do trato urinário, reduzido ganho de peso materno, diabetes gestacional, prematuridade, baixo peso ao nascer e complicações no parto (AZEVEDO et al., 2015; SOUSA et al., 2013).

Considerando este contexto e a necessidade de maior conhecimento sobre o assunto, este artigo de revisão narrativa da literatura tem como objetivo discorrer sobre as intercorrências obstétricas mais comuns em mulheres adolescentes.

\section{METODOLOGIA}

Trata-se de um artigo de revisão narrativa da literatura, que utilizou como fonte para a pesquisa bibliográfica as bases de dados eletrônicas SciELO e PubMed. Realizou-se a seleção de artigos utilizando como descritores "gravidez na adolescência" e "complicações na gravidez".

Foram incluídos artigos científicos de ensaios clínicos, estudos observacionais, experimentais e revisões de literatura, disponíveis para download na íntegra, na versão em Português, publicados no período de 2010 a 2021 e que se relacionavam com gravidez na adolescência e intercorrências obstétricas. Foram excluídos série de casos, relato de casos, relato de experiência e editoriais, assim como também foram excluídos artigos que não estavam relacionados ao objetivo do trabalho e artigos que foram encontrados em ambas as bases de dados.

\section{FUNDAMENTAÇÃO TEÓRICA}

Foram identificados 365 artigos, sendo 346 provenientes da base de dados SciELO e 19 da base de dados PubMed. Após a aplicação dos critérios de exclusão, foram selecionados 35 artigos para a revisão de literatura, sendo estes considerados os mais relevantes para a formulação da discussão.

Para o diagnóstico de uma gravidez, faz-se necessário a utilização da história clínica, exame físico e exames laboratoriais, após suspeita, na ocorrência de amenorreia ou atraso menstrual (MENEGATTI; OLIVEIRA; GAMA, 2014). A gestação é um acontecimento natural e fisiológico, entretanto algumas situações são classificadas como gestações de risco, por apresentarem condições que aumentam a probabilidade de complicações durante esta fase (PINTO et al., 2020). Uma dessas condições é a gravidez no período da adolescência, pois o sistema reprodutivo das mulheres ainda está passando por modificações, com o início do aparecimento dos caracteres sexuais secundários, como as mamas e os pelos pubianos, e até os primeiros ciclos ovulatórios, apresentando imaturidade física para sustentar uma gestação com segurança (RIBEIRO et al., 2017). 
Entre as intercorrências obstétricas comuns na gestação na adolescência, pode-se citar as doenças hipertensivas, síndromes hemorrágicas, infecção do trato urinário, anemia, abortos, baixo peso ao nascer e parto prematuro (SILVA et al., 2021).

O baixo peso ao nascer tem íntima relação com as condições físicas da gestante, e associado à prematuridade são os principais fatores que levam ao aumento da mortalidade neonatal (COSTA; SENA; DIAS, 2011). É considerado baixo peso o neonato que apresenta, ao nascer, peso de até 2500 gramas. Esta condição pode levar a problemas na vida da criança no futuro (SURITA et al., 2011).

A pré-eclâmpsia e a eclampsia são consideradas doenças hipertensivas da gestação e ambas têm como fator de risco a hipertensão arterial crônica. Estão entre as complicações mais comuns da gestação, e podem levar a um elevado número de mortes maternas (SOUSA et al., 2013).

Muitas das complicações obstétricas estão mais associadas às condições de vida materna, bem como o início tardio do pré-natal, falta de apoio familiar e condições de vulnerabilidade social (DIAS; TEIXEIRA, 2010). As adolescentes que se encontram durante uma gestação necessitam de atenção especializada durante o pré-natal e também após o parto, com a finalidade de promover a saúde da gestante e do concepto, e identificar os possíveis riscos advindos da gravidez nesta fase da vida (SOUSA et al., 2013).

\section{CONSIDERAÇÕES FINAIS}

A gravidez na adolescência é um problema de saúde da população brasileira, gerando complicações físicas para a mãe e para o bebê, além de também apresentar consequências psicossociais para todos os envolvidos. Entre as intercorrências obstétricas comuns na gestação na adolescência, destacam-se a pré-eclâmpsia e a eclampsia, infecções urinárias, parto prematuro e baixo peso ao nascer. Com a realização de um pré-natal adequado, com uma equipe multidisciplinar, tais complicações podem ser prevenidas, por isso é importante divulgar o conhecimento sobre as intercorrências obstétricas nas gestações na adolescência à equipe multiprofissional que dá assistência às adolescentes durante o pré-natal.

\section{PRINCIPAIS REFERÊNCIAS}

AZEVEDO, Walter Fernandes de et al. Complicações da gravidez na adolescência: revisão sistemática da literatura. São Paulo, Einstein, v. 13, n. 14, p. 618-626, 2015.

COSTA, Evaldo Lima da; SENA, Maria Cristina Ferreira; DIAS, Adriano. Gravidez na adolescência - determinante para prematuridade e baixo peso. Brasília, Comunicação em Ciências da Saúde, v. 22 , n.1, p. 183-188, 2011.

DIAS, Ana Cristina Garcia; TEIXEIRA, Marco Antônio Pereira. Gravidez na adolescência: um olhar sobre um fenômeno complexo. Ribeirão Preto, Paidéia, v. 20, n. 45, p. 123-131, 2010. 
MARTINS, Marília da Glória et al. Associação de gravidez na adolescência e prematuridade. São Paulo, Revista Brasileira de Ginecologia e Obstetrícia, v. 33, n. 11, p. 354-360, 2011.

MENEGATTI, Luciana; OLIVEIRA, Rafael Bosco de; GAMA, Ivson Lelis. Complicações da gravidez na adolescência. Colider, Facider Revista Científica, n. 6, p. 17-31, 2014.

OLIVEIRA, Elaine Fernandes Viellas de; GAMA, Silvana Granado Nogueira da; SILVA, Cosme Marcelo Furtado Passos da. Gravidez na adolescência e outros fatores de risco para mortalidade fetal e infantil no município do Rio de Janeiro, Brasil. Rio de Janeiro, Caderno de Saúde Pública, v. 26, n. 3, p. 567-578, 2010.

OYAMADA Luiz Henrique et al. Gravidez na adolescência e o risco para a gestante. Paraná, Brazilian Journal of Surgery and Clinical Research - BJSCR, v. 6, n. 2, p. 38-45, 2014.

PINTO, Kelly Cristina de Lima Ramos et al. Principais complicações gestacionais e obstétricas em adolescentes. Curitiba, Brazilian Journal of Health Review, v. 3, n. 1, p. 873-882, 2020.

RIBEIRO, José Francisco et al. Complicações obstétricas em adolescente atendidas em uma maternidade pública de referência. Recife, Revista de Enfermagem UFPE Online, v. 11. n. 7, p. 2728-2735, 2017.

SILVA, Isabelle Oliveira Santos da et al. Intercorrências obstétricas na adolescência e a mortalidade materna no Brasil: uma revisão sistemática. Curitiba, Brazilian Journal of Health Review, v.4, n. 2 , p. 6720-6734, 2021.

SOUSA, Aretuza Seixas de et al. Complicações obstétricas em adolescentes de uma maternidade. Recife, Revista de Enfermagem UFPE Online, v. 7, n. 4, p. 1167-1173, 2013.

SURITA, Fernanda Garanhani Castro et al. Fatores associados ao baixo peso ao nascimento entre adolescentes no Sudeste do Brasil. São Paulo, Revista Brasileira de Ginecologia e Obstetrícia, v. 33, n. 10, p. 286-291, 2011. 